\title{
Epulis géante pédiculée : à propos d'un cas
}

\section{Giant pediculate epulis: a case report}

CHARLES BENGONDO MESSANGA (1), JULES ONANA (2), ZACHARIE SANDO (3), XAVIER EBOLO (2), GENÉVIÈVE BENGONO (1)

\section{RÉSUMÉ}

Les auteurs rapportent le cas rare d'une épulis géante, de $12 \times 10 \mathrm{~cm}$, observée chez une patiente de 23 ans. La tumeur qui prenait son origine dans les alvéoles déshabitées de 36 et 37, était responsable d'une disgrâce physique. Longtemps traitée en milieu traditionnel, la patiente est arrivée tardivement à l'hôpital, avec une volumineuse tumeur de consistance élastique encombrant la cavité buccale, occasionnant une gêne fonctionnelle et faisant craindre un risque d'obstruction pharyngée à moyen terme. Pour des raisons économiques, l'intervention a été effectuée sous anesthésie loco-régionale ; elle a été longue et difficile du fait de l'encombrement buccal et du saignement. Elle a consisté à réaliser une section du pédicule de l'épulis, une dénudation de l'os, une révision des alvéoles, puis un débridement étendu des tissus périphériques pathologiques. Les suites ont été simples, permettant à la patiente de retrouver la joie de vivre. L'analyse histopathologique la pièce opératoire a conclu qu'il s'agissait d'une épulis, sans aucun signe de transformation maligne. (Med Buccale Chir Buccale 2006; 12: 159-62).

mots clés : épulis, tumeur bénigne, chirurgie, tradipraticien

médecine buccale chirurgie buccale

VOL. $12, \mathrm{~N}^{\circ} 3$ 2006

page 159

\section{SUMMARY}

The authors report a rare case of a giant epulis, measuring $12 \mathrm{~cm} \times 10 \mathrm{~cm}$, observed on a 23 years old patient. The tumour is disgraceful, taking root into the sockets of teeth 36 and 37 extracted. The patient has been treated for a long time in the traditional milieu, and she arrived late to the hospital. This great tumour of flexible consistency occupied the whole mouth and induced functional discomfort and risk of pharyngeal obstruction at middle time. Because the patient had not enough money, the surgical intervention has been done under locoregional anaesthesia. The operation was difficult and had a long duration because of the mouth congestion and bleeding. The intervention began by cutting epulis root and then a bone denudation, a review of the socket and extensive debridement of pathological tissue were done. The postoperation period was simple and allowed the patient to find the living joyful.

The essential pathology analysis of surgical material confirmed the absence of malignancy. (Med Buccale Chir Buccale 2006; 12: 159-62).

key words : epulis, benign tumour, surgery, tradipractitioner

1- Département ORL-Ophtalmologie-Stomatologie, Faculté de Médecine et des Sciences Biomédicales, Université de Yaoundé I.

2- Service d'Odonto-Stomatologie, Hôpital Militaire de Yaoundé.

3- Département Anatomie Pathologique, Faculté de Médecine et des Sciences Biomédicales, Université de Yaoundé I.

Demande de tirés à part :

Dr BENGONDO MESSANGA Charles, Département ORL-Ophtalmologie-Stomatologie, Faculté de Médecine et des Sciences Biomédicales, Université de Yaoundé I, Cameroun. e-mail : bengondo@yahoo.fr 
médecine buccale chirurgie buccale

VOL. $12, \mathrm{~N}^{\circ} 3$ 2006

page 160
L'épulis, tumeur bénigne inflammatoire des gencives, secondaire à une épine irritative in situ ${ }^{[1]}$ est observée à tout âge. Cette tumeur, plus fréquente chez la femme [2,3], siège habituellement sur le versant vestibulaire de la région incisivo-canine, plus rarement dans la région molaire. Parfois, elle se développe entre deux dents, entrainant leur déplacement, l'apparition d'une mobilité et d'une alvéolyse, ou dans une alvéole déshabitée. L'épulis est souvent sessile, parfois pédiculée, de consistance élastique, de couleur rouge vineux dans la forme inflammatoire aiguë. La muqueuse peut être normale, mamelonnée ou ulcérée. Cette inflammation peut survenir au cours d'une gingivite chronique de cause locale, chez la femme enceinte, ou au cours de troubles hématologiques ${ }^{[4,5]}$.

Malgré les variétés histologiques (inflammatoire, angiomateuse, à cellules géantes, fibreuse, fibroblastique), le diagnostic est assez facile et la prise en charge chirurgicale aisée, car le volume tumoral reste en général modéré. Non traitée, l'épulis peut prendre des dimensions importantes, occasionnant une gêne fonctionnelle et un préjudice esthétique non négligeables.

L'objectif de cet article est de présenter une épulis de taille inhabituelle, et son traitement dans un contexte particulier et dans un service d'Odontostomatologie sous-équipé.

\section{OBSERVATION}

Une jeune femme, âgée de 23 ans, est venue consulter dans le service d'Odontostomatologie de I'hôpital militaire de Yaoundé (Cameroun) pour une volumineuse tumeur jugale gauche. L'histoire de la maladie commence deux ans auparavant par des douleurs dentaires banales sur 36 et 37 . Après échec de l'auto-médication (amoxicilline et ibuprofène), la patiente a recours au traitement traditionnel (décoctions diverses, scarifications, incantations...), sans plus de succès. Devant l'augmentation progressive de la tumeur jugale, le tradipraticien aurait affirmé qu'il s'agissait d'un sort lancé par un proche parent et qu'il ne fallait surtout pas accepter une intervention à l'hôpital, qui pourrait être fatale. Démoralisée, la jeune fille est entrée dans un groupe de prières pour tenter de conjurer le sort ; ce fut un nouvel échec. La tuméfaction évoluait vers l'oro-pharynx et occupait plus des $2 / 3$ du vestibule, entraînant une gêne fonctionnelle de plus en plus importante. Sur les conseils insistants de ses voisins et de ses amis qui lui répétaient que si elle ne mourrait pas des suites d'une éventuelle opération, elle allait mourir de la tumeur elle-même, la patiente s'est enfin décidée à consulter dans le service.

La patiente, mère d'un nourrisson de trois mois, ne présentait aucun antécédent médico-chirurgical particulier, et son état général n'était pas altéré. Son passage chez les tradipraticiens et le sentiment de ne jamais pouvoir guérir, suscitaient une profonde angoisse avec d'importantes répercussions psychiques.

L'examen exo-buccal montrait une asymétrie faciale due à une volumineuse tuméfaction, intéressant toute l'hémiface gauche (Fig. 1). II n'y avait pas d'adénopathies submandibulaires ou cervicales palpables. A l'examen endo-buccal, on observe une tumeur implantée dans les alvéoles déshabitées 36 et 37. De couleur rouge vineux et de consistance élastique, elle occupait plus des $2 / 3$ du vestibule gauche et débordait largement du côté lingual, constituant un risque d'obstruction pharyngée à moyen terme. La muqueuse recouvrant la tumeur était d'aspect normal excepté les zones de contact avec les dents supérieures où elle était ulcérée (Fig. 2). La gêne fonctionnelle était très importante. Un bilan biologique pré-opératoire et un orthopantomogramme ont été réalisés. Les résultats du bilan

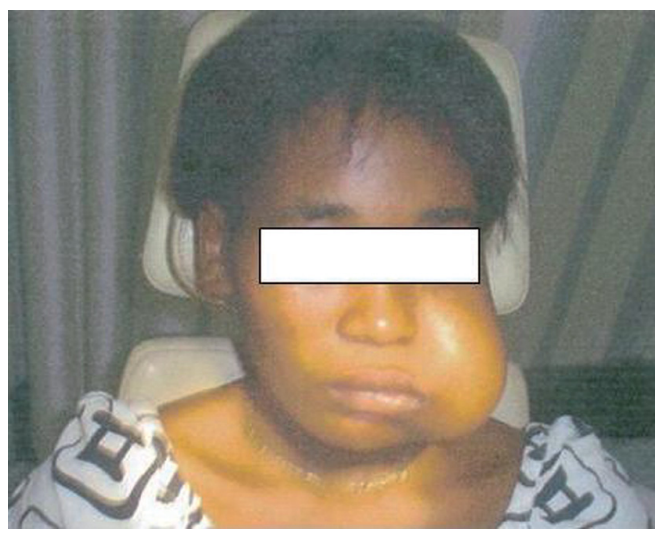

Figure 1 : Tuméfaction jugale gauche avec importante asymétrie faciale.

Left jugal tumefaction with an important facial asymetrie. 


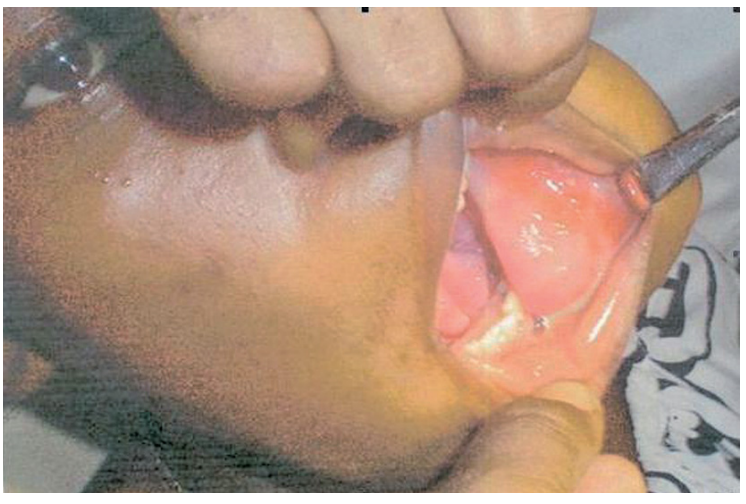

Figure 2 : Vue endo-buccale montrant l'épulis. Intra-oral aspect showing the epulis.

sont arrivés après deux mois et ne révélaient rien de particulier ; la radiographie montrait une alvéolyse en regard de la base d'implantation du pédicule tumoral.

En raison des difficultés économiques, l'intervention a été faite sous anesthésie loco-régionale, complétée par des infiltrations à la base de la tumeur avec de la xylocaïne à $2 \%$ et de l'adrénaline. L'intervention, très difficile en raison des conditions et de la taille tumorale, a consisté en une section du pédicule, puis une dénudation de l'os et un débridement étendu du tissu pathologique. Après avoir excisé la totalité de la tumeur avec son pédicule, une révision alvéolaire a été effectuée. La pièce opératoire mesurait $12 \times 10 \mathrm{~cm}$ (Fig. 3). Faute de bistouri électrique pour cautériser la plaie, le saignement a été abondant au cours de l'intervention. II a été contrôlé par des sutures et une injection intramusculaire d'étamsylate (250 mg) suivie d'une prescription per os (respectivement 500 mg. $3 \times \mathrm{x}^{-1} \times 3 \mathrm{j}$ ).

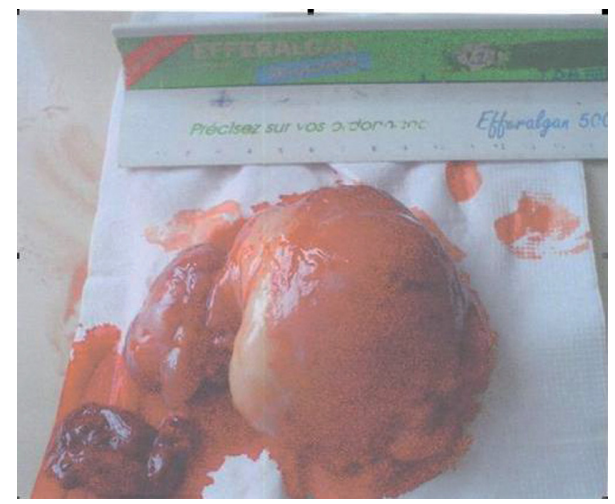

Figure 3 : Epulis réséquée. Epulis after resection.
Après l'intervention, la patiente a présenté une lipothymie qui a nécessité une hospitalisation d'une journée pour observation. Une association spiramycine et métronidazole (500 mg et $1 \mathrm{~g} \mathrm{j}^{-1} \times 10 \mathrm{j}$ ) a été prescrite ainsi que du diclofénac (150 $\mathrm{mg}^{\mathrm{j}^{-1} \mathrm{x}}$ 5 j) et de l'hexétidine en bains de bouche. Les suites opératoires ont été simples. Une semaine plus tard la patiente avait retrouvé un aspect normal et la joie de vivre (Fig 4). L'examen histopathologique de la pièce opératoire a confirmé l'absence de malignité et montré qu'elle était constituée de tissu conjonctif inflammatoire, hyperplasique, avec une abondante néo-vascularisation ; cet aspect est caractéristique d'une épulis inflammatoire.

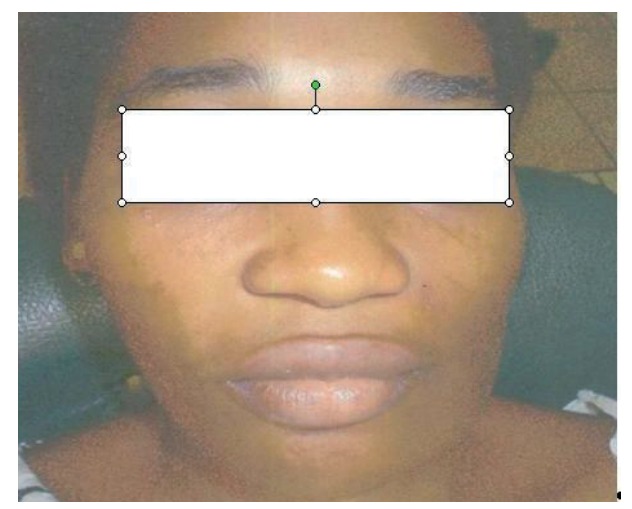

médecine

buccale chirurgie buccale

VOL. $12, \mathrm{~N}^{\circ} 3$ 2006

page 161
Figure 4 : Aspect post-opératoire de la patiente. Post surgical aspect of the patient.

\section{DISCUSSION}

L'épulis est une hyperplasie bénigne de la gencive qui s'observe plus souvent chez la femme, surtout pendant la grossesse $[2,6,7]$. II s'agit d'une tumeur très fréquente qui atteint rarement la taille de celle rapportée dans cette observation. Ce gigantisme serait lié à la conjugaison de plusieurs facteurs : retard de prise en charge en milieu hospitalier, action irritante du traumatisme répété dû aux dents supérieures, réalisation de scarifications et application de diverses mixtures par les tradipraticiens et surtout modifications endocriniennes liées à la grossesse. Abraham et al [7] et Armitage ${ }^{[6]}$ ont montré que ces modifications hormonales favorisent le développement des épulis. Les tradipraticiens ont certes une action positive dans quelques affections mais ils dépassent sou- 
médecine buccale chirurgie buccale

VOL. $12, \mathrm{~N}^{\circ} 3$ 2006

page 162 vent le champ de leurs compétences. Pour eux, toute pathologie se résume à un sort jeté par une tierce personne. Ils déconseillent vivement aux patients d'aller à l'hôpital, sous peine d'y mourir ${ }^{[8]}$. Le plus souvent, leur comportement contribue à aggraver la maladie, principalement en retardant sa prise en charge médicale. A cela, s'ajoutent l'ignorance et la pauvreté de la population.

Malgré la taille inhabituelle de cette épulis, le diagnostic différentiel avec une tumeur maligne (carcinome épidermoïde...) s'est fait sur la base de la lenteur de l'évolution, l'absence d'adénopathies, l'absence de saignement spontané et la mobilité de la tumeur. Le diagnostic a été confirmé par l'examen histopathologique de la pièce opératoire.

L'intervention chirurgicale s'est révélée longue et complexe en raison des conditions opératoires et de l'encombrement buccal lié à la taille de l'épulis. II a fallu aussi également faire face à un important saignement per-opératoire qui aurait été plus facilement contrôlé avec un bistouri électrique ${ }^{\left[{ }^{9}\right.}$. Les suites opératoires ont été simples, certainement en raison de la compliance et de l'hygiène bucco-dentaire rigoureuse de la patiente.

\section{CONCLUSION}

L'épulis inflammatoire, ou granulome pyogénique, est une tumeur bénigne fréquente due à une hyperplasie localisée de la gencive, de nature inflammatoire, secondaire à la cicatrisation imparfaite d'une lésion gingivale. Cette cicatrisation anormale peut être aggravée par des irritations locales et des facteurs endocriniens. La pauvreté, l'ignorance, la difficulté d'accès aux centres hospitaliers modernes, l'itinéraire thérapeutique erratique (tradipraticiens, groupe de prières...) sont autant de facteurs retardant la prise en charge de nombreuses affections aboutissant à un préjudice esthétique et psychosocial (exclusion...) parfois très important. Les centres primaires de santé doivent jouer pleinement leur rôle dans le dépistage et l'orientation des patients afin d'accélérer leur prise en charge médicale.

\section{RÉFÉRENCES}

1 - ACHARD JL. Tumeurs bénignes de la muqueuse buccale (p 151-158). In Révision accélérée en odonto-stomatologie. Maloine Paris, 1988.

2 - Le Breton G. Pathologie de la muqueuse buccale (p 349-403). In Traité de sémiologie et clinique odontostomatologique. Cdp, Vélizy, 1997.

3 - PeUCH-Lestrade GR. A propos d'une épulis inflammatoire. Chir Dent 2004 ; 1183 : 77-8.

4 - Glicman I. Parodontologie clinique. Julien Prélat, Paris, 1974.

5 - Dechaume M, Grellet M, Laudenbach P, Payen J. Précis de Stomatologie, $5^{\circledR}$ éd. Masson, Paris, 1980.

6 - ARMITAGE GC. Periodontal disease and pregnancy: discussion, conclusion and recommandation. Ann Periodontol $2001 ; 6$ : 189-92.
7 - Abraham-Impijn l, Polsacheva DV, Raber DuRlaker JE. The signification of endocrine factors and micro-organisms in development of gingivitis in pregnant woman. Stomatologica $1996 ; 75: 15-8$

8 - Bengondo CH, Bitha T, Ngoa S, Fewou A, Bengono G. Ostéite nécrosante de l'angle mandibulaire avec asymétrie faciale secondaire à une extraction dentaire. A propos d'un cas. Odonto-Stomatol Tropicale 2001; 1996: 30-34.

9 - BeAUPERE L. Bistouri électrique. Encycl Med Chir Paris, Stomatologie I, 22020 A10, 1983 